\title{
A Multi-Wavelength Study of the Oxygen-Rich AGB Star CIT 3: Bispectrum speckle interferometry and dust-shell modelling
}

\author{
K.-H. Hofmann, T. Blöcker, G. Weigelt \\ Max-Planck-Institut für Radioastronomie, 53121 Bonn, Germany \\ Y. Balega
}

Special Astrophysical Observatory, Nizhnij Arkhyz, 35147, Russia

CIT 3 is an oxygen-rich long-period variable evolving along the Asymptotic Giant Branch and is one of the most extreme infrared AGB objects. Due to substantial mass loss it is surrounded by an optically thick dust shell which absorbs almost all visible light radiated by the star and finally re-emits it in the infrared regime. We present the first near infrared bispectrum speckle-interferometry observations of CIT 3 in the $J-, H$-, and $K^{\prime}$-band (resolution: 48 mas, 56 mas, and 73 mas). The interferograms were obtained with the Russian SAO $6 \mathrm{~m}$ telescope. While CIT 3 appears almost spherically symmetric in the $H$ - and $K^{\prime}$-band it is clearly elongated in the $J$-band along a symmetry axis of position angle $-28^{\circ}$. Two structures can be identified: a compact elliptical core (eccentricity $\sim 0.8$ ) and a fainter north-western fan-like structure (full opening angle $\sim 40^{\circ}$ ). The development of such asphericities close to the central star suggests that CIT 3 is in the very end of its AGB evolution or even in transition to the proto-planetary nebula phase where most objects are observed in axisymmetric geometry.

Extensive radiative transfer calculations have been carried out and confronted with the spectral energy distribution ranging from $1 \mu \mathrm{m}$ to $1 \mathrm{~mm}$, our $1.24 \mu \mathrm{m}, 1.65 \mu \mathrm{m}$ and $2.12 \mu \mathrm{m}$ visibility functions, as well as $11 \mu \mathrm{m}$ ISI interferometry. The best model found to match the observations refers to a cool central star with $T_{\text {eff }}=2250 \mathrm{~K}$ which is surrounded by an optically thick dust shell with $\tau(0.55 \mu m)=30$. The central-star diameter is 10.9 mas and the inner dust shell diameter 71.9 mas, being in line with lunar occultation observations. The inner dust-shell rim is located at $r_{1}=6.6 R_{*}$ and has a temperature of $T_{1}=900 \mathrm{~K}$. A two-component model existing of an inner uniform-outflow shell region $\left(\rho \sim 1 / r^{2}, r<20.5 r_{1}\right)$ and an outer region where the density declines more shallow as $\rho \sim 1 / r^{1.5}$ proved to give the best overall match of the observations. Provided the outflow velocity kept constant, the more shallow density distribution in the outer shell indicates that mass-loss has decreased with time in the past of CIT 3. Adopting $v_{\exp }=20 \mathrm{~km} / \mathrm{s}$, the termination of that mass-loss decrease and the begin of the uniform-outflow phase took place $87 \mathrm{yr}$ ago. The present-day mass-loss rate can be determined to be $\dot{M}=(1.3-2.1) \cdot 10^{-5} \mathrm{M}_{\odot} / \mathrm{yr}$ for $d=500-800 \mathrm{pc}$ (Hofmann et al. 2001, A\&A 379, 529). 